\title{
Association analysis of genetic variants in the ghrelin and tumor necrosis factor $\alpha$ genes and the risk for non-Hodgkin's lymphoma in Kuwaitis
}

\author{
Maryam H. Alrashid ${ }^{\mathrm{a}, *}$, Ahmad Al-Serri ${ }^{\mathrm{b}}$, Salem H. Alshemmaric, ${ }^{\mathrm{c}}$, Jeethu Anu Geo ${ }^{\mathrm{a}}$ and \\ Suzanne A. Al-Bustan ${ }^{\mathrm{a}}$ \\ ${ }^{a}$ Department of Biological Sciences, Faculty of Science, Kuwait University, Safat, Kuwait \\ ${ }^{\mathrm{b}}$ Department of Pathology, Unit of Human Genetics, Faculty of Medicine, Kuwait University, Safat, Kuwait \\ ${ }^{\mathrm{c}}$ Department of Medicine, Faculty of Medicine, Kuwait University, Safat, Kuwait \\ ${ }^{\mathrm{d}}$ Kuwait Cancer Control Center, Kuwait City, Kuwait
}

Received 14 May 2019

Accepted 29 April 2021

\begin{abstract}
.
BACKGROUND: Non-Hodgkin's lymphoma (NHL) is the most common hematological malignancy in the world. Many etiologic factors have been implicated in the risk of developing NHL, including genetic susceptibility and obesity. Single-nucleotide polymorphisms (SNPs) in Ghrelin (GHRL), an anti-inflammatory hormone, and tumor necrosis factor $\alpha(T N F-\alpha)$, an inflammatory cytokine, have been independently associated with the risk for obesity and NHL.

OBJECTIVE: To investigate the association between SNPs in GHRL and TNF- $\alpha$ and the risk for NHL and obesity in Kuwaitis. METHODS: We recruited 154 Kuwaiti NHL patients and 217 controls. Genotyping was performed for rs 1629816 (GHRL promoter region), rs35684 (GHRL 3' untranslated region), and rs1800629 (TNF- $\alpha$ promoter region). Logistic regression analysis was performed to assess the association of the investigated SNPs with NHL and the relationship between the selected SNPs with BMI in each group separately.

RESULTS: We show that rs 1629816 GG was associated with an increased risk for NHL in our sample $(p=0.0003$, OR 1.82; CI: 1.31-2.54). None of the investigated SNPs were associated with obesity, nor was obesity found to be associated with the risk for NHL.

CONCLUSIONS: Our study demonstrates an association between rs1629816, a SNP in the GHRL regulatory region, and NHL in Kuwaitis.
\end{abstract}

Keywords: SNPs, GHRL, TNF- $\alpha$, non-Hodgkin's lymphoma

\section{Introduction}

Non-Hodgkin's lymphoma (NHL) is the most common hematological malignancy in the world [14]. In Kuwait and the Gulf Council Countries (GCC), NHL

\footnotetext{
${ }^{*}$ Corresponding author: Maryam H. Alrashid, Department of Biological Sciences, Faculty of Science, Kuwait University, PO Box 5969, Safat 13060, Kuwait. Tel.: +965 24987086 24985649; Fax: +965 24847054; E-mail: maryam.alrashid@ku.edu.kw.
}

is the fifth most common cancer in both males and females [14]. The NHL incidence rate in Kuwait has increased at least 1.5 times in the last 33 years [12].

Various etiologic factors contribute to the risk of NHL, the major ones being genetic susceptibility, chronic inflammation and lifestyle factors, such as obesity $[2,11,13]$. Genetic association studies have shown associations between NHL and specific polymorphisms, especially in genes that have been implicated in inflammatory processes [45]. 
Studies have reported conflicting results on the link between NHL and obesity, with some studies showing a positive association while others showing no association $[22,28,47]$. Kuwait has one of the highest obesity rates in the world. The prevalence of overweight and obesity in the Kuwaiti population are $80.4 \%$ and $47.5 \%$, respectively, and are higher in females, $81.9 \%$ and $53 \%$, compared to males, $78 \%$ and $39.2 \%$ [1]. Obesity is associated with chronic low-grade inflammation because adipocytes produce and secrete several proinflammatory cytokines, including tumor necrosis factor alpha (TNF$\alpha$ ) [46]. The $T N F$ - $\alpha$ single-nucleotide polymorphism (SNP) rs1800629 (TNF- $\alpha-308 \mathrm{G} / \mathrm{A})$, is a polymorphism that leads to increased production of TNF- $\alpha$ [48]. The association of rs 1800629 with obesity is inconsistent and varies based on ethnicity and sex [35]. On the other hand, this SNP has been associated with an increased risk for numerous cancers [7,18,29,32,36]. In the International Lymphoma Epidemiology Consortium study, rs 1800629 was found to be significantly associated with the risk for NHL [42]. Other studies found that this association might be population-specific; the risk of NHL in carriers of the rs 1800629 variant allele was found to be either significantly increased $[19,53]$, decreased or absent depending on the population studied [51].

TNF- $\alpha$ inhibits ghrelin (GHRL), an anti-inflammatory hormone and a growth hormone (GH) secretagogue that is released mainly during caloric restriction and weight loss [17], and GHRL inhibits proinflammatory cytokine production, including TNF- $\alpha[25,33]$. TNF- $\alpha$ is inversely related to GHRL's expression and activity, and reduced levels of GHRL have been observed with increased circulating levels of TNF- $\alpha$, such as in the chronic inflammation state of obese individuals $[39,43]$. It has been reported that obese individuals have lower levels of GHRL in their plasma compared with individuals with a normal body mass index (BMI) $[6,8]$. A reduction in the GHRL level is associated with increased inflammation during obesity, and increased GHRL level induced by caloric restriction is associated with decreased inflammation [8]. Several studies have investigated the association of polymorphisms in the GHRL gene with BMI and reported contradictory results, with most studies reaching the conclusion that they are not associated [10,30,44].

Recent studies have shown that GHRL stimulates cancer cell proliferation and metastasis and is highly expressed in several cancers, including NHL [26,41]. However, meta-analysis studies have been inconclusive about whether SNPs in GHRL were associated with cancer, including NHL $[9,37]$. One study showed that two GHRL SNPs, rs1629816 (GHRL-4427G>A) in the promoter region and $\mathrm{rs} 35684(\mathrm{GHRL}+5179 \mathrm{~A}>\mathrm{G})$ in the 3' untranslated region, were associated with a significantly decreased risk for NHL [44]. Interestingly, neither SNP causes a change in the amino acid that it encodes, and therefore, both are considered synonymous SNPs (sSNPs) [31,44].

Since polymorphisms of the $G H R L$ and $T N F-\alpha$ genes have been associated with obesity and NHL risk in different populations and since this association has never been investigated in the Kuwaiti population, we aimed to determine the genotypic and allelic frequencies of GHRL rs 1629816 and rs35684, and the TNF- $\alpha$ rs 1800629 in a sample of healthy Kuwaiti individuals and NHL patients and to investigate the potential association of these polymorphisms with the risk of developing NHL. We also examined whether these polymorphisms were associated with obesity in our cohort and whether obesity was associated with NHL.

\section{Materials and methods}

\subsection{Study cohorts}

We recruited a total of 217 controls and 154 NHL patients for this study. The controls were cancer-free individuals recruited from polyclinics and matching as well as possible the patient group for sex and age. The NHL patients were diagnosed and recruited at the Kuwait Cancer Care Center (KCCC). Upon arrival at the clinic, a blood sample was collected $(5 \mathrm{ml})$ into a sterile EDTA vacutainer and stored at $4^{\circ} \mathrm{C}$ until further processing for DNA extraction. Clinical data collected from the patients consisted of an in-clinic interview and review of the patient medical records when needed. Sex, age, weight, and height were recorded (Table 1). Out of the 154 patients, 139 patients were included in the study. The exclusion criteria were patients who did not provide informed consent or a blood sample at the time of the interview and those who were non-Kuwaitis.

\subsection{Ethical approval and informed consent}

Ethical approval for this study was obtained in 2012 from the Joint Committee for The Protection of $\mathrm{Hu}$ man Subjects in Research of the Health Sciences Center (HSC) and the Kuwait Institute of Medical Specialization (KIMS)-Ministry of Health-Kuwait, following the guidelines set by the The Code of Ethics of the World Medical Association (Declaration of Helsinki, 1964). All patients who participated in the study provided an informed consent. 
Table 1

Demographics and clinical features of the study groups

\begin{tabular}{lccc}
\hline Characteristics of the study sample & Controls $(n=217)$ & NHL Patients $(n=139)$ & $p$ \\
\hline BMI & $28.8 \pm 6.7$ & $27.8 \pm 7.5$ & 0.19 \\
Male, $n(\%)$ & $123(56.7 \%)$ & $67(48.2 \%)$ & 0.47 \\
Age, $y$ & $50.7 \pm 15.6$ & $53.9 \pm 15$ & 0.79 \\
Age of onset, $y$ & - & $51.5 \pm 14.9$ & - \\
Treatment regimen for NHL patients & $95(68.3 \%)$ & \\
Chemotherapy & $1(0.7 \%)$ & \\
Radiotherapy & $21(15.1 \%)$ & \\
Chemotherapy + radiotherapy & $2(1.4 \%)$ & \\
Bone marrow transplant & $11(7.9 \%)$ & & \\
No treatment & $9(6.5 \%)$ & & \\
No information & & \\
\hline
\end{tabular}

Values given are the mean \pm standard deviation or number of patients $(\mathrm{N})(\%)$. Body mass index (BMI), non-Hodgkin's lymphoma (NHL).

Table 2

Location and reference numbers of SNPs included in this study

\begin{tabular}{lccl}
\hline rs number & Gene & Polymorphism & SNP location \\
\hline rs1800629 & TNF- $\alpha$ & $-308 \mathrm{G}>\mathrm{A}$ & Promoter \\
rs35684 & GHRL & 5179A $>\mathrm{G}$ & 3' UTR region \\
rs1629816 & GHRL & $-4427 \mathrm{G}>\mathrm{A}$ & Promoter \\
\hline
\end{tabular}

RefSNP (rs) numbers represent the SNP's accession number in the SNP database (dbSNP). Untranslated region (UTR).

\subsection{Genotyping}

Total genomic DNA was extracted from blood samples using the Gentra ${ }^{\circledR}$ Puregene ${ }^{\circledR}$ DNA extraction Kit according to the manufacturer's instructions (Qiagen Hilden, Germany). Genotyping by allelic discrimination using real-time PCR was performed on all samples for all three investigated SNPs (Table 2) on the ABI PRISM 7900HT Fast Real-Time PCR System (Life Technologies Co. Carlsbad CA, USA). Initial optimization of genotyping assays for the various SNP loci was performed by employing custom-designed or published primers that are annotated in the GenBank database reference sequence NG_007462.1 [20,40,42,44]. The protocol conditions used were those described and published for Taqman ${ }^{\circledR}$ genotyping assays. Samples were genotyped for each polymorphism as homozygous or heterozygous.

\subsection{Quality assurance of SNP detection}

Quality assurance was performed as described previously [3]. In brief, SNP sites were sequenced to verify the accuracy of the real-time PCR allele calling using custom primers that were designed in the lab using NCBI Primer-Blast and Primer 3 software. For each locus, samples were randomly selected (10 samples per $\mathrm{SNP}$ ), and the target regions (flanking sequences of the variant sites not exceeding $500 \mathrm{bp}$ ) were sequenced for confirmation by Sanger sequencing on the ABI Gene Analyzer 3130xl using the Big Dye Terminator Kit v3.1 with conditions according to the manufacturer's instructions (Applied Biosystems, Life Technologies, CA, USA). Confirmed samples were then used as positive controls in RT-PCR reactions. Sequences for rs35684 and rs1800629 were submitted to GenBank under accession numbers KP339513 and KP339511, respectively.

\subsection{Statistical analysis}

Hardy-Weinberg equilibrium for each SNP was tested using a web-based Pearson's chi-square test calculator. Statistical analysis was performed to assess the relationship of each SNP with susceptibility to developing NHL using logistic regression. The results are reported as odds ratios (ORs) and $95 \%$ confidence intervals (CIs). In addition, linear regression analysis was performed to assess the relationship between the selected SNPs and BMI in each group separately. Age, sex and BMI were controlled for in the regression analysis. Data were analyzed using SPSS (Version 25; SPSS, Inc., an IBM Company, Chicago, Illinois). Demographic data and characteristics are represented as the mean \pm standard deviation (SD) and percentage, as appropriate. Statistical significance was accepted at $p<0.05$.

\section{Results}

\subsection{Demographics and clinical characteristics}

Two hundred seventeen healthy controls (123 males, 94 females) and 139 NHL patients (67 males, 72 females) were recruited for this study. The mean age of 
Table 3

Genotypic and allelic frequency of the $T N F-\alpha$ SNP rs1800629 and the GHRL SNPs rs35684 and rs1629816 in controls $(n=217)$ vs. patients $(n=139)$

\begin{tabular}{lccc}
\hline SNP & Control $N(\%)$ & NHL patients $N(\%)$ & $p$ \\
\hline$T N F-\alpha$ & & & \\
rs1800629 & & $98(70.5 \%)$ & 0.671 \\
GG & $162(74.5 \%)$ & $32(23 \%)$ & \\
GA & $44(20 \%)$ & $9(6.5 \%)$ & \\
AA & $11(5.5 \%)$ & & \\
Ghrelin & & $76(54.5 \%)$ & 0.562 \\
rs35684 & & $54(39 \%)$ & \\
AA & $106(49 \%)$ & $9(6.5 \%)$ & \\
AC & $95(44 \%)$ & & \\
CC & $16(7 \%)$ & $56(40.3 \%)$ & $\mathbf{0 . 0 0 2}$ \\
rs1629816 & & $66(47.5 \%)$ & \\
GG & $62(28.5 \%)$ & $17(12.2 \%)$ & \\
GA & $97(45 \%)$ & $58(26.5 \%)$ & \\
AA & & & \\
\hline
\end{tabular}

Values given are the number of individuals $(\mathrm{N})(\%)$.

Table 4

HWE for the $T N F-\alpha$ SNP rs 1800629 and the GHRL SNPs rs 35684 and rs1629816 in controls $(n=217)$ vs. patients $(n=139)$

\begin{tabular}{lcc}
\hline SNP & HWE & Non-HWE \\
\hline RS 1629816 & $(p=0.11)$ & - \\
Control & $(p=0.72)$ & - \\
Patient & $(p=0.40)$ & - \\
RS 35684 & $(p=0.89)$ & - \\
Control & - & $(p=0.002)$ \\
Patient & - & $(p=0.01)$ \\
RS 1800629 & - & \\
Control & Patient &
\end{tabular}

Hardy-Weinberg Equilibrium (HWE): if $p<0.05$, then the SNP is not in HWE.

the controls and the patients was $50.7 \pm 15.6$ and 53.9 \pm 15 years, respectively. There were no significant differences between healthy controls and NHL patients in terms of BMI, sex or age (Table 1).

\subsection{Genotype distributions and allele frequencies}

The investigated SNPs and their locations in their respective genes are presented in Table 2 . The allelic and genotypic frequencies of the three SNPs are summarized in Table 3 . The allelic frequencies for the various genotypes (Table 4) were found to be in HardyWeinberg equilibrium (HWE), except for rs1800629 in patients, controls and the whole sample $(p<0.05)$. Of the alleles, the most frequent wild-type allele expressed in both the controls and patients was that of rs1800610, while the most frequent variant allele expressed was that of rs1629816 (Table 3). There was a significant difference in the genotype frequency between the control and the patients for rs1629816 $(p=0.002)$, but not for
Table 5

Multivariate analysis to predict variables associated with developing NHL in the patient $(n=139)$ cohort

\begin{tabular}{lll}
\hline Variable & \multicolumn{1}{c}{ OR $(95 \% \mathrm{CI})$} & \multicolumn{1}{c}{$p$} \\
\hline Gender (female) & $1.15(0.73-1.83)$ & 0.53 \\
Age $(+1$ year $)$ & $1.01(0.99-1.02)$ & 0.166 \\
BMI $\left(\mathrm{kg} / \mathrm{m}^{2}\right)$ & $0.97(0.94-1)$ & 0.122 \\
GHRL $r \mathrm{~s} 1629816$ & $1.82(1.31-2.54)$ & $\mathbf{0 . 0 0 0 3}$ \\
\hline
\end{tabular}

rs 1800629 ( $p=0.671)$ or rs35684 ( $p=0.562)$. All investigated genotypic variants were observed in both the control and patient samples (Table 3). Only one of the investigated SNPs, rs1629816, showed a significant difference in its allelic and genotypic distribution between the patients and the control samples.

\subsection{Association of rs 1629816 with $N H L$}

Analysis by logistic regression showed a strong association of rs1629816 GG genotype with the risk of developing NHL in our sample ( $p=0.0003$, OR, 1.82; 95\% CI, 1.31-2.54, Table 5). All associations were controlled for age, sex and body mass index (BMI). We also analyzed the association of nongenetic parameters with NHL. We found that in our study cohort, age, obesity or sex showed no association with the risk for NHL (Table 5, $p>0.05$ ). Finally, we analyzed the association of the investigated SNPs with BMI but found no association in the controls or the patients (data not shown, $p>0.05$ ).

\section{Discussion}

In our study, we have investigated the association of SNPs in the GHRL and TNF- $\alpha$ genes with the risk for NHL in a group of Kuwaiti individuals. We also looked into whether these SNPs were associated with obesity and whether obesity was associated with an increased risk for NHL in our study cohort. We found that rs 1629816, a SNP in the GHRL promoter, was associated with an increased risk for NHL in carriers of the GG genotype $(p=0.0003$, OR, 1.82 ; 95\% CI, 1.312.54 , Table 5), which is consistent with what was previously reported [44]. The association of GHRL polymorphism with NHL is plausible for several reasons. GHRL is an anti-inflammatory hormone that has been found to have an association with many cancers, including NHL. It is thought to give cells a growth advantage because of its effect on $\mathrm{GH}$ and therefore cell proliferation. GHRL has also been found to inhibit apoptosis through the extracellular signal-regulated kinase $1 / 2$ 
Table 6

Distribution of $T N F-\alpha$ rs 1800629 genotypes in different populations

\begin{tabular}{lccccc}
\hline & Kuwait (\%) & Asian (\%) & Non Hispanic (\%) & Persian (\%) & Egypt (\%) \\
\hline Control & & & & & \\
& GG 74.5 & GG 85.3 & GG 71 & GG 81.3 & GG 67 \\
& GA 20.0 & GA 14 & GA 17.1 & GA 17.1 & GA 27 \\
& AA 5.5 & AA 0.7 & AA 1.6 & AA 1.6 & AA 6 \\
NHL & & & & & \\
& GG 70.5 & GG 88.1 & GG 68 & GG 85.3 & GG 44.8 \\
& GA 23 & GA 11.4 & GA 28 & GA 13.2 & GA 25 \\
& AA 6.5 & AA 0.5 & AA 4 & AA 1.5 & AA 26.2 \\
\hline
\end{tabular}

(ERK1/2) and phosphatidylinositol-4,5-bisphosphate 3-kinase (PI3K)/Akt protein kinase B (PKB) pathways [49,50,54]. The ERK1/2 and PI3K/Akt pathways are staple oncogenic pathways that have been established to be upregulated in several cancers. Increased cell proliferation and inhibition of apoptosis are both hallmarks of the process of carcinogenesis [16]. Therefore, it is no surprise that GHRL has been found to be abnormally expressed in many cancers. The most accepted reason for the association between GHRL SNPs and the risk for NHL is that variation in GHRL alters its expression and/or function, thereby increasing its effect on cell proliferation and/or inhibiting apoptosis, which in turn may stimulate lymphomagenesis, leading to an increased risk of developing NHL [43]. The SNP associated with NHL risk in our sample, rs1629816, is a sSNP in GHRL. sSNPs can occur in coding and noncoding regions of a gene. Studies show that SSNPs affect protein expression, conformation and function and thereby may lead to altered disease susceptibilities $[23,31]$. This has been shown to be true in various diseases, including hematological and nonhematological malignancies [31]. rs1629816 might affect GHRL expression, conformation or function, leading to an increase in its effect on cancer cell proliferation and therefore an individual's susceptibility to various malignancies, including NHL. However, further studies are needed to elucidate the effect of this SSNP on GHRL and the molecular mechanism by which it might be increasing the risk for NHL.

We also looked into the $T N F-\alpha$ SNP rs 1800629 and found no difference in the genotypic distribution of this SNP between controls and NHL patients in our sample $(p=0.671)$; therefore, there was no link between rs1800629 and the risk of NHL in our sample. This is consistent with a previous meta-analysis in which the $T N F$ - $\alpha$ SNP rs 1800629 GG was associated with an increased risk for NHL in Caucasians, decreased risk in Asians, but no association when the populations were combined [51].
In our sample, none of the investigated SNPs were associated with an obese BMI $(p>0.05$, data not shown), nor was BMI associated with the risk for NHL ( $p=$ 0.122, OR 0.97 95\% CI, 0.94-1, Table 5). Studies associating NHL with overweight or obesity have had conflicting results. A study in Canada that included more than 20,000 individuals with 19 different cancers found a $46 \%$ increase in the risk for NHL in people with a BMI above $30 \mathrm{~kg} / \mathrm{m}^{2}$ [5]. Conversely, a study of 37,931 women in Iowa found no association between BMI and NHL [5,38].

All tested SNPs were in HWE except for rs1800629. This SNP is of particular importance since it has been shown to be associated with an increased risk for several chronic conditions, such as asthma, chronic obstructive pulmonary disease (in Asian but not Caucasian populations), and several cancers $[4,15,24,27,52]$. Several studies on rs 1800629 and the risk for NHL in different populations found it to deviate from HWE: in controls in Seattle, in a combined sample of controls and patients in the UK, and in the control groups, the patient groups, and the whole samples in Spain and at the University of California-San Francisco [42]. Quality control testing showed no genotyping errors in these studies, and other SNPs tested in the same populations were consistently found to be in HWE. The same applies for our sample; stringent quality control measures, sequencing of SNPs and random replication of the genotyping test for already tested samples were applied to our samples and confirmed the accuracy of our genotyping. In addition, other SNPs tested in this study and a previous one were always in HWE [3]. This precludes the possibility of genotyping error and warrants further investigation into the pattern of this SNP's distribution across different populations. We found that the allelic frequency of this SNP has differed among different populations (Table 5); the frequency of the A allele was found to be highest in Egyptians (0.2), followed by non-Hispanic whites and Kuwaitis (0.16), Persians (0.1) and finally Asians (0.08) [19,21,34,42]. The association of the risk of NHL with rs1800629 was highest in Egypt, followed 
by non-Hispanic whites, while no risk was reported for Persians or Kuwaitis (this study), and a negative association was reported for Asians [19,21,34,42]. Interestingly, we noticed that the degree of risk association might correlate with the frequency of the A allele in a population, although no conclusions can be drawn until additional studies investigate this observation.

Although larger than some previous case-control studies, a limitation of our study is the sample size. This is a common limitation in genetic studies due to the difficulties surrounding the availability of samples. Although NHL is the top hematological malignancy in Kuwait, collecting a sufficient number of samples remains a significant challenge since a very small number of samples are available for collection due to the small population size. Increasing the sample size might give us better insight into the relationship of the risk of NHL with the $T N F$ - $\alpha$ SNP rs1800629.

In conclusion, our study shows that rs1629810, a SNP in GHRL promoter area, was highly associated with the risk of NHL in our study cohort. None of the SNPs investigated were associated with obesity, and obesity was also not associated with the risk of developing NHL. Future studies including a higher number of subjects and from different populations are necessary to validate this association. Studies are also needed to identify genes whose SNPs are associated with NHL to help predict individuals who are at risk and therefore offer the benefit of early detection or even to apply preventative measures where appropriate. Finally, relating our population to other populations in terms of SNP genotype and allele frequency will be helpful for taking advantage of future pharmacogenomic applications that are based on individual and population genotypes.

\section{Acknowledgments}

The authors would like to acknowledge the support of Kuwait University Research Sector (Grant SL02/11) and the General Facility Project (GS 01/02) for the use of the ABI 3130xl Gene Analyzer. The authors extend their deepest appreciation and gratitude to all of the participants in this study, as well as the staff at Shiekha Badriya Alsabah Medical Oncology and Stem Cell Transplant Centre, Kuwait Cancer Control Centre, Kuwait City, Kuwait, for their assistance with the blood collection. The PI would also like to thank Miss Rubina Fatima for her technical assistance in organizing the collected data.

\section{Conflict of interest}

The authors declare no conflicts of interest.

\section{Author contributions}

Conception: M.H. Alrashid, S.H. Alshemmari, S.A. Al-Bustan.

Interpretation or analysis of data: M.H. Alrashid, A. Al-Serri, J.A. Geo, S.A. Al-Bustan.

Preparation of the manuscript: M.H. Alrashid, A. AlSerri, S.A. Al-Bustan.

Revision for important intellectual content: M.H. Alrashid, A. Al-Serri, S.H. Alshemmari, J.A. Geo, S.A. Al-Bustan.

Supervision: M.H. Alrashid, S.A. Al-Bustan.

\section{References}

[1] I. Al Rashdan and Y. Al Nesef, Prevalence of overweight, obesity, and metabolic syndrome among adult Kuwaitis: results from community-based national survey, Angiology 61 (2010), 42-8.

[2] D.D. Alexander, P.J. Mink, H.O. Adami, E.T. Chang, P. Cole, J.S. Mandel and D. Trichopoulos, The non-Hodgkin lymphomas: a review of the epidemiologic literature, Int J Cancer 120(Suppl 12) (2007), 1-39.

[3] M.H. Alrashid, A. Al-Serri, S.H. Alshemmari, P. Koshi and S.A. Al-Bustan, Association of Genetic Polymorphisms in the VKORC1 and CYP2C9 Genes with Warfarin Dosage in a Group of Kuwaiti Individuals, Mol Diagn Ther 20 (2016), 183-90.

[4] T. Aoki, T. Hirota, M. Tamari, K. Ichikawa, K. Takeda, T. Arinami, M. Shibasaki and E. Noguchi, An association between asthma and TNF-308G/A polymorphism: meta-analysis, J Hum Genet 51 (2006), 677-85.

[5] J.R. Cerhan, C.A. Janney, C.M. Vachon, T.M. Habermann, N.E. Kay, J.D. Potter, T.A. Sellers and A.R. Folsom, Anthropometric characteristics, physical activity, and risk of nonHodgkin's lymphoma subtypes and B-cell chronic lymphocytic leukemia: a prospective study, Am J Epidemiol 156 (2002), 527-35.

[6] D.E. Cummings, D.S. Weigle, R.S. Frayo, P.A. Breen, M.K. Ma, E.P. Dellinger and J.Q. Purnell, Plasma ghrelin levels after diet-induced weight loss or gastric bypass surgery, $N$ Engl $J$ Med 346 (2002), 1623-30.

[7] F.E. Davies, S.J. Rollinson, A.C. Rawstron, E. Roman, S Richards, M. Drayson, J.A. Child and G.J. Morgan, Highproducer haplotypes of tumor necrosis factor alpha and lymphotoxin alpha are associated with an increased risk of myeloma and have an improved progression-free survival after treatment, J Clin Oncol 18 (2000), 2843-51.

[8] V.D. Dixit, Adipose-immune interactions during obesity and caloric restriction: reciprocal mechanisms regulating immunity and health span, J Leukoc Biol 84 (2008), 882-92. 
[9] L. Dossus, J.D. McKay, F. Canzian, S. Wilkening, S. Rinaldi, C. Biessy, A. Olsen, A. Tjonneland, M.U. Jakobsen, K. Overvad, F. Clavel-Chapelon, M.C. Boutron-Ruault, A. Fournier, J. Linseisen, A. Lukanova, H. Boeing, E. Fisher, A. Trichopoulou, C. Georgila, D. Trichopoulos, D. Palli, V. Krogh, R. Tumino, P. Vineis, J.R. Quiros, N. Sala, C. MartinezGarcia, M. Dorronsoro, M.D. Chirlaque, A. Barricarte, F.J. van Duijnhoven, H.B. Bueno-de-Mesquita, C.H. van Gils, P.H. Peeters, G. Hallmans, P. Lenner, S. Bingham, K.T. Khaw, T.J. Key, R.C. Travis, P. Ferrari, M. Jenab, E. Riboli and R. Kaaks, Polymorphisms of genes coding for ghrelin and its receptor in relation to anthropometry, circulating levels of IGF-I and IGFBP-3, and breast cancer risk: a case-control study nested within the European Prospective Investigation into Cancer and Nutrition (EPIC), Carcinogenesis 29 (2008), 1360-6.

[10] H. Du, K.S. Vimaleswaran, L. Angquist, R.D. Hansen, A.D. van der, C. Holst, A. Tjonneland, K. Overvad, M.U. Jakobsen, H. Boeing, K. Meidtner, D. Palli, G. Masala, N. Bouatia-Naji, W.H. Saris, E.J. Feskens, N.J. Wareham, T.I. Sorensen and R.J. Loos, Genetic polymorphisms in the hypothalamic pathway in relation to subsequent weight change - the DiOGenes study, PLoS One 6 (2011), e17436.

[11] K. Ekstrom-Smedby, Epidemiology and etiology of nonHodgkin lymphoma - a review, Acta Oncol 45 (2006), 258-71.

[12] A. Elbasmi, A. Al-Asfour, Y. Al-Nesf and A. Al-Awadi, Cancer in Kuwait: magnitude of the problem, Gulf J Oncolog 1 (2010), $7-14$.

[13] A.M. Evens and B.C. Chiu, The challenges of epidemiologic research in non-Hodgkin lymphoma, JAMA 300 (2008), 205961.

[14] J. Ferlay, M. Ervik, F. Lam, M. Colombet, L. Mery, M. Piñeros, A. Znaor, I. Soerjomataram and F. Bray, Global cancer observatory: cancer today, 2018.

[15] X.F. Guo, J. Wang, S.J. Yu, J. Song, M.Y. Ji, Z. Cao, J.X Zhang, J. Wang and W.G. Dong, TNF-alpha-308 polymorphism and risk of digestive system cancers: A meta-analysis, World J Gastroenterol 19 (2013), 9461-71.

[16] D. Hanahan and R.A. Weinberg, Hallmarks of cancer: the next generation, Cell 144 (2011), 646-74.

[17] H. Himmerich and A.J. Sheldrick, TNF-alpha and ghrelin: opposite effects on immune system, metabolism and mental health, Protein Pept Lett 17 (2010), 186-96.

[18] S.Y. Ho, Y.J. Wang, H.L. Chen, C.H. Chen, C.J. Chang, P.J. Wang, H.H. Chen and H.R. Guo, Increased risk of developing hepatocellular carcinoma associated with carriage of the TNF2 allele of the -308 tumor necrosis factor-alpha promoter gene, Cancer Causes Control 15 (2004), 657-63.

[19] H.D. Hosgood, 3rd, W.Y. Au, H.N. Kim, J. Liu, W. Hu, J. Tse, B. Song, K.F. Wong, J.J. Lee, S.J. Chanock, L.P. Siu, M.P. Purdue, M.H. Shin, J. Yu, R. Liang, H.J. Kim, N. Rothman and Q. Lan, IL10 and TNF variants and risk of non-Hodgkin lymphoma among three Asian populations, Int J Hematol 97 (2013), 793-9.

[20] https://www.ncbi.nlm.nih.gov/genbank/.

[21] A. Ibrahim, H. Abdel Rahman, M. Khorshied, R. Sami, N. Nasr and O. Khorshid, Tumor necrosis factor alpha-308 and Lymphotoxin alpha+252 genetic polymorphisms and the susceptibility to non-Hodgkin lymphoma in Egypt, Leuk Res 36 (2012), 694-8.

[22] S.C. Larsson and A. Wolk, Body mass index and risk of non-Hodgkin's and Hodgkin's lymphoma: A meta-analysis of prospective studies, Eur J Cancer 47 (2011), 2422-30.

[23] A. Lazrak, L. Fu, V. Bali, R. Bartoszewski, A. Rab, V. Havasi, S. Keiles, J. Kappes, R. Kumar, E. Lefkowitz, E.J. Sorscher, S.
Matalon, J.F. Collawn and Z. Bebok, The silent codon change I507-ATC->ATT contributes to the severity of the DeltaF508 CFTR channel dysfunction, FASEB J 27 (2013), 4630-45.

[24] H. Li, Z. Jin, X. Li, L. Wu and J. Jin, Associations between single-nucleotide polymorphisms and inflammatory bowel disease-associated colorectal cancers in inflammatory bowel disease patients: a meta-analysis, Clin Transl Oncol 19 (2017), 1018-1027.

[25] W.G. Li, D. Gavrila, X. Liu, L. Wang, S. Gunnlaugsson, L.L. Stoll, M.L. McCormick, C.D. Sigmund, C. Tang and N.L. Weintraub, Ghrelin inhibits proinflammatory responses and nuclear factor-kappaB activation in human endothelial cells, Circulation 109 (2004), 2221-6.

[26] T.C. Lin and M. Hsiao, Ghrelin and cancer progression, Biochim Biophys Acta Rev Cancer 1868 (2017), 51-57.

[27] N. Liu, G.J. Liu and J. Liu, Genetic association between TNFalpha promoter polymorphism and susceptibility to squamous cell carcinoma, basal cell carcinoma, and melanoma: A metaanalysis, Oncotarget 8 (2017), 53873-53885.

[28] A. Lopez-Suarez, Burden of cancer attributable to obesity, type 2 diabetes and associated risk factors, Metabolism 92 (2019), 136-146.

[29] J.C. Machado, C. Figueiredo, P. Canedo, P. Pharoah, R. Carvalho, S. Nabais, C. Castro Alves, M.L. Campos, L.J. Van Doorn, C. Caldas, R. Seruca, F. Carneiro and M. SobrinhoSimoes, A proinflammatory genetic profile increases the risk for chronic atrophic gastritis and gastric carcinoma, Gastroenterology 125 (2003), 364-71.

[30] G.R. Martin, J.C. Loredo and G. Sun, Lack of association of ghrelin precursor gene variants and percentage body fat or serum lipid profiles, Obesity (Silver Spring) 16 (2008), 908-12.

[31] C. McCarthy, A. Carrea and L. Diambra, Bicodon bias can determine the role of synonymous SNPs in human diseases, BMC Genomics 18 (2017), 227.

[32] S. Mestiri, N. Bouaouina, S.B. Ahmed, A. Khedhaier, B.B. Jrad, S. Remadi and L. Chouchane, Genetic variation in the tumor necrosis factor-alpha promoter region and in the stress protein hsp70-2: susceptibility and prognostic implications in breast carcinoma, Cancer 91 (2001), 672-8.

[33] T. Narula and B.P. deBoisblanc, Ghrelin in Critical Illness, $A m$ J Respir Cell Mol Biol 53 (2015), 437-42.

[34] H. Nasiri, S. Farajnia, A. Rezamand, A.A. Movassaghpour H.A. Esmaeili, A. Monfaredan, N. Mobarra, N. Rahimifar, L. Sahebi and M. Farshdousti Hagh, Genetic Variations of Tumor Necrosis Factor -alpha-308 and Lymphtoxin-alpha+252 in Non-Hodgkin Lymphoma and Acute Lymphoblastic Leukemia Patients, Iran J Basic Med Sci 16 (2013), 990-5.

[35] Z.Y. Ng, M.K. Veerapen, W.M. Hon and R.L. Lim, Association of leptin/receptor and TNF-alpha gene variants with adolescent obesity in Malaysia, Pediatr Int 56 (2014), 689-97.

[36] B.R. Oh, M. Sasaki, G. Perinchery, S.B. Ryu, Y.I. Park, P. Carroll and R. Dahiya, Frequent genotype changes at -308, and 488 regions of the tumor necrosis factor-alpha (TNF-alpha) gene in patients with prostate cancer, J Urol 163 (2000), 15847 .

[37] N.A. Pabalan, I. Seim, H. Jarjanazi and L.K. Chopin, Associations between ghrelin and ghrelin receptor polymorphisms and cancer in Caucasian populations: a meta-analysis, BMC Genet 15 (2014), 118.

[38] S.Y. Pan, K.C. Johnson, A.M. Ugnat, S.W. Wen, Y. Mao and G. Canadian, Cancer Registries Epidemiology Research, Association of obesity and cancer risk in Canada, Am J Epidemiol 159 (2004), 259-68.

[39] J. Pereira, F.C. da Silva and P.M.M. de Moraes-Vieira, The 
Impact of Ghrelin in Metabolic Diseases: An Immune Perspective, J Diabetes Res 2017 (2017), 4527980.

[40] M.P. Purdue, L.C. Sakoda, B.I. Graubard, R. Welch, S.J. Chanock, I.A. Sesterhenn, M.V. Rubertone, R.L. Erickson and K.A. McGlynn, A case-control investigation of immune function gene polymorphisms and risk of testicular germ cell tumors, Cancer Epidemiol Biomarkers Prev 16 (2007), 77-83.

[41] S. Sever, D.L. White and J.M. Garcia, Is there an effect of ghrelin/ghrelin analogs on cancer? A systematic review, Endocr Relat Cancer 23 (2016), R393-409.

[42] C.F. Skibola, P.M. Bracci, A. Nieters, A. Brooks-Wilson, S. de Sanjose, A.M. Hughes, J.R. Cerhan, D.R. Skibola, M. Purdue, E. Kane, Q. Lan, L. Foretova, M. Schenk, J.J. Spinelli, S.L. Slager, A.J. De Roos, M.T. Smith, E. Roman, W. Cozen, P. Boffetta, A. Kricker, T. Zheng, T. Lightfoot, P. Cocco, Y. Benavente, Y. Zhang, P. Hartge, M.S. Linet, N. Becker, P. Brennan, L. Zhang, B. Armstrong, A. Smith, R. Shiao, A.J. Novak, M. Maynadie, S.J. Chanock, A. Staines, T.R. Holford, E.A. Holly, N. Rothman and S.S. Wang, Tumor necrosis factor (TNF) and lymphotoxin-alpha (LTA) polymorphisms and risk of non-Hodgkin lymphoma in the InterLymph Consortium, $\mathrm{Am}$ J Epidemiol 171 (2010), 267-76.

[43] C.F. Skibola, J.D. Curry and A. Nieters, Genetic susceptibility to lymphoma, Haematologica 92 (2007), 960-9.

[44] D.R. Skibola, M.T. Smith, P.M. Bracci, A.E. Hubbard, L. Agana, S. Chi and E.A. Holly, Polymorphisms in ghrelin and neuropeptide $\mathrm{Y}$ genes are associated with non-Hodgkin lymphoma, Cancer Epidemiol Biomarkers Prev 14 (2005), 12516.

[45] S.S. Wang and A. Nieters, Unraveling the interactions between environmental factors and genetic polymorphisms in non-Hodgkin lymphoma risk, Expert Rev Anticancer Ther 10 (2010), 403-13.

[46] K. Warzocha, P. Ribeiro, J. Bienvenu, P. Roy, C. Charlot, D. Rigal, B. Coiffier and G. Salles, Genetic polymorphisms in the tumor necrosis factor locus influence non-Hodgkin's lymphoma outcome, Blood 91 (1998), 3574-81.

[47] E.V. Willett, L.M. Morton, P. Hartge, N. Becker, L. Bernstein, P. Boffetta, P. Bracci, J. Cerhan, B.C. Chiu, P. Cocco, L. Dal
Maso, S. Davis, S. De Sanjose, K.E. Smedby, M.G. Ennas, L. Foretova, E.A. Holly, C. La Vecchia, K. Matsuo, M. Maynadie, M. Melbye, E. Negri, A. Nieters, R. Severson, S.L. Slager, J.J. Spinelli, A. Staines, R. Talamini, M. Vornanen, D.D. Weisenburger, E. Roman and C. Interlymph, Non-Hodgkin lymphoma and obesity: a pooled analysis from the InterLymph Consortium, Int J Cancer 122 (2008), 2062-70.

[48] A.G. Wilson, J.A. Symons, T.L. McDowell, H.O. McDevitt and G.W. Duff, Effects of a polymorphism in the human tumor necrosis factor alpha promoter on transcriptional activation, Proc Natl Acad Sci U S A 94 (1997), 3195-9.

[49] Y. Xiang, Q. Li, M. Li, W. Wang, C. Cui and J. Zhang, Ghrelin inhibits AGEs-induced apoptosis in human endothelial cells involving ERK1/2 and PI3K/Akt pathways, Cell Biochem Funct 29 (2011), 149-55.

[50] X. Zeng, S. Chen, Y. Lin and Z. Ke, Acylated and unacylated ghrelin inhibit apoptosis in myoblasts cocultured with colon carcinoma cells, Oncol Rep 39 (2018), 1387-1395.

[51] K. Zhai, J. Ding and Y. Zhou, Different role of tumor necrosis factor-alpha polymorphism in non-Hodgkin lymphomas among Caucasian and Asian populations: a meta-analysis, Int J Mol Sci 15 (2014), 7684-98.

[52] S. Zhang, C. Wang, B. Xi and X. Li, Association between the tumour necrosis factor-alpha-308G/A polymorphism and chronic obstructive pulmonary disease: an update, Respirology 16 (2011), 107-15.

[53] Y. Zhang, M.Y. Wang, J. He, J.C. Wang, Y.J. Yang, L. Jin, Z.Y Chen, X.J. Ma, M.H. Sun, K.Q. Xia, X.N. Hong, Q.Y. Wei and X.Y. Zhou, Tumor necrosis factor-alpha induced protein 8 polymorphism and risk of non-Hodgkin's lymphoma in a Chinese population: a case-control study, PLoS One 7 (2012), e37846.

[54] Y. Zhang, B. Ying, L. Shi, H. Fan, D. Yang, D. Xu, Y. Wei, X. Hu, Y. Zhang, X. Zhang, T. Wang, D. Liu, L. Dou, G. Chen, F. Jiang and F. Wen, Ghrelin inhibit cell apoptosis in pancreatic beta cell line HIT-T15 via mitogen-activated protein kinase/phosphoinositide 3-kinase pathways, Toxicology 237 (2007), 194-202. 